\title{
Primary immunodeficiency disease: a retrospective study of 112 Chinese children in a single tertiary care center
}

Jinhong Wu, Wenwei Zhong, Yong Yin and Hao Zhang

\begin{abstract}
Background: Primary immunodeficiency disease (PID) is a disorder caused by an inherited flaw in the immune system that increases the susceptibility to infections.

Methods: In this study, 112 children with PID were diagnosed and classified based on the 2017 criteria presented by the International Union of Immunological Societies (IUIC) in a single tertiary care center from January 2013 to November 2018. We retrospectively studied the clinical features of those PID children and followed-up them as well.

Results: It was revealed that male/female ratio was 6:1. The most frequent diagnosed PID was severe combined immunodeficiency (SCID) (28.6\%) and hyper-IgM (HIGM) syndrome (24.1\%), followed by predominantly antibody deficiencies (17.8\%). Combined immunodeficiencies with associated or syndromic features (12.5\%) and congenital defects of phagocyte number, function, or both (10.7\%) were less common in our center compared with SCID and HIGM syndrome. Besides, we found that 20 children (17.8\%) had a positive family history of PID, and almost all cases (97.3\%) had a history of recurrent infection. Recurrent respiratory tract infection was among the most common symptoms, followed by the bacterial infection of the skin and mucous membranes and diarrhea. Additionally, adverse event following immunization (AEFI) was found in $20.5 \%$ of the patients, and immune disorder was commonly observed in PID patients. In the present study, 47 patients underwent allogeneic hematopoietic stem cell transplantation (allo-HSCT), and 2-year overall survival (OS) rate for these patients was 78.7\% (37/47). It is noteworthy that OS widely differed among PID patients with different phenotypes who underwent allo-HSCT. The 2-year OS rate for SCID, HIGM syndrome, and the remaining of PID patients who underwent allo-HSCT was 14.3, 83.3, and 100\%, respectively.
\end{abstract}

Conclusions: PID typically emerges at early age. Recurrent infection and serious infection were the most common clinical manifestations. Allo-HSCT is a relatively effective therapeutic strategy for PID patients.

Keywords: Primary immunodeficiency disease, Clinical features, Demographic characteristics, Children, China

\section{Background}

Primary immunodeficiency disease (PID) refers to a heterogeneous group of over 130 disorders that result from defects in immune system development and/or function. With the development and use of flow cytometry and genetic technology for clinical diagnosis, diagnostic rate of PID has been markedly increased in China. To date, 354 distinct disorders with 344 different gene defects have been identified in patients with PID [1]. PID is characterized by diverse clinical manifestations, such as

\footnotetext{
* Correspondence: zhang123hao2004@163.com

Department of Pulmonary, Shanghai Children's Medical Center Affiliated to

Shanghai Jiaotong University School of Medicine, Shanghai 200127, China
}

recurrent or prolonged serious infections, autoimmune/ inflammatory disease, allergy, or malignancy. In addition, physicians or general practitioners may not able to recognize PID because of rarity of those diseases, particularly in developing countries (e.g., China) [2, 3]. As a result, delayed diagnosis or misdiagnosis are quite common in clinical practice, which may lead to the poor outcomes for children with PID. In the present study, we retrospectively analyzed the clinical features and prognosis of 112 PID children over a 6-year period in a single tertiary care center to strengthen our understanding about PID.

(c) The Author(s). 2019 Open Access This article is distributed under the terms of the Creative Commons Attribution 4.0 International License (http://creativecommons.org/licenses/by/4.0/), which permits unrestricted use, distribution, and reproduction in any medium, provided you give appropriate credit to the original author(s) and the source, provide a link to the Creative Commons license, and indicate if changes were made. The Creative Commons Public Domain Dedication waiver (http://creativecommons.org/publicdomain/zero/1.0/) applies to the data made available in this article, unless otherwise stated. 


\section{Methods}

\section{Classification and diagnosis}

A single-center retrospective study was carried out at Shanghai Children's Medical Center Affiliated to Shanghai Jiao Tong University (Shanghai, China) from January 2013 to November 2018. A total of 112 patients were included for investigation and classified according to the 2017 criteria presented by the International Union of Immunological Societies (IUIS) classification system [1]. In addition, PID was grouped as follows: immunodeficiencies affecting cellular and humoral immunity; predominantly antibody-deficiency diseases; combined immunodeficiencies with associated or syndromic features; diseases of immune dysregulation; congenital defects of phagocyte number or function; defects in intrinsic and innate immunity; autoinflammatory disorders; and complement deficiencies $[4,5]$. Because the clinical features and prognosis of hyper-IgM (HIGM) syndrome are different from those associated with severe combined immunodeficiency (SCID), patients with HIGM syndrome were not herein included in the group of immunodeficiencies influencing cellular and humoral immunity. All patients were diagnosed according to the diagnostic criteria and confirmed by genetic phenotyping. Immunodeficiencies secondary to other conditions (e.g., human immunodeficiency virus (HIV) infection) were excluded as well.

\section{Data collection}

All clinical data were collected from the hospital medical records. Sex, family history, age of the first hospitalization, clinical features, onset of symptoms, signs, age of onset, age at diagnosis, auxiliary examination, and disease progress were included in this study.

\section{Specimen collection and sequencing analysis of relevant genes}

Patients' specimens were collected during hospitalization from January 2013 to November 2018. For comparative purposes, we also collected specimens from the parents. Anticoagulant venous blood was collected and was further centrifuged at $3500 \mathrm{rpm}$ for $10 \mathrm{~min}$. Peripheral blood cells were stored at $-80^{\circ} \mathrm{C}$ and used for DNA extraction and gene sequencing using next-generation sequencing (NGS) technique, which were further performed based on the clinical features and auxiliary examination results.

\section{Statistical analysis}

Data were analyzed by using SPSS 17.0 software (IBM, Armonk, NY, USA). The differences between the groups were compared by the Student's $t$-test. $P$ values $<0.05$ were considered statistically significant.

\section{Results}

Demographic features of PID patients

Frequency and distribution of PID

Diagnosis was based on clinical, immunological, and genetic testing, and 112 patients were diagnosed with PID in our hospital from January 2013 to November 2018. There were 18 PIDs diagnosised in 112 patients with pathogenic mutations which grouped into 7 main categories (Table 1). As shown in Table 1, SCID was found in 32 patients (28.6\%), HIGM syndrome in 27 (24.1\%), combined immunodeficiencies with associated or syndromic features $14(12.5 \%)$, predominantly antibody deficiencies in 20 (17.8\%), diseases related to immune dysregulation in 4 (3.6\%), congenital defects of phagocyte number or function, or both in $12(10.7 \%)$, defects in intrinsic and innate immunity in $2(1.8 \%)$, and complement deficiencies in $1(0.9 \%)$ (Table 1$)$. None of the patients were diagnosed with phenocopies of PID or defects in autoinflammatory disorders. Combination of T- and B-cell immunodeficiencies was the most common $(n=32)$ among all the patients with PID, followed by HIGM syndrome $(n=27)$. Wiskott-Aldrich syndrome (WAS) was the most common PID in combined immunodeficiencies with associated or syndromic features group $(n=9)$, followed by dyskeratosis congenita $(n=2)$. Predominantly antibody deficiencies were the most common PIDs in patients with severe serum immunologlobulin isotypes reduction and $\mathrm{B}$ cells decreased or absent $(n=16)$, followed by common variable immunodeficiency disorders $(n=3)$. There were 7 and 5 patients who diagnosed with chronic granulomatous disease and congenital neutropenias, respectively. Those patients showed congenital defects in phagocyte number, function or both.

\section{Patients' demographic characteristics}

There were 96 males and 16 females in the present study. The male/female ratio was $6: 1$, and there were different ratios among subgroups of PIDs (Table 2). All patients with PID related to X-linked recessive inheritance were male (e.g., Bruton tyrosine kinase (BTK)-deficiency, WAS, and HIGM syndrome).

\section{Distribution of age in patients with PID}

The average onset age of all patients with PID was 13 months (range, 2 days- 117 months). The onset age of 68 children $(60.7 \%)$ was younger than 6 months, there were 28 (25\%) patients aged between 6 months and 3 years, and $16(14.3 \%)$ patients were older than 3 years as well. The average time to the first diagnosis as PID was 24 months (range, 1-130 months). Besides, 38 (33.9\%) children were diagnosed with PID who were younger than 6 months, 45 (40.2\%) children aged between 6 months and 3 years, and 29 (25.9\%) children were older than 3 years (Table 2). There were statistically significant 
Table 1 Distribution of PIDs according to the International Union of Immunological Societies criteria

\begin{tabular}{|c|c|}
\hline Type & No. of Cases (\%) \\
\hline Immunodeficiencies affecting cellular and humoral immunity & $59(28.6)$ \\
\hline SCID & 32 \\
\hline HIGM & 27 \\
\hline Combined immunodeficiencies with associated or syndromic features & $14(12.5)$ \\
\hline WAS & 9 \\
\hline DKC & 2 \\
\hline DGS & 1 \\
\hline EDA-ID & 1 \\
\hline HIES & 1 \\
\hline Predominantly antibody deficiencies & $20(17.8)$ \\
\hline Severe reduction in all serum immunologlobulin isotypes with profoundly decreased or absent B cells & 16 \\
\hline CVID & 3 \\
\hline APDS & 1 \\
\hline Diseases of immune dysregulation & $4(3.6)$ \\
\hline IPEX syndrome & 2 \\
\hline Immune dysregulation with colitis & 1 \\
\hline CHS & 1 \\
\hline Congenital defects of phagocyte number or function & $12(10.7)$ \\
\hline CGD & 7 \\
\hline Congenital neutropenias & 5 \\
\hline Defects in intrinsic and innate immunity & $2(1.8)$ \\
\hline IFN- $\gamma$ receptor deficiency & 1 \\
\hline Predisposition to invasive fungal diseases (CARD9 deficiency) & 1 \\
\hline Complement deficiencies & $1(0.9)$ \\
\hline Complement deficiencies & 1 \\
\hline
\end{tabular}

Abbreviations: APDS activated PI3K syndrome immunodeficiency, CGD chronic granulomatous disease, CHS Chediak-Higashi syndrome, CVID common variable immunodeficiency disorders, DGS DiGeorge syndrome, DKC dyskeratosis congenital, EDA-ID anhidrotic ectodermodysplasia with immunodeficiency, HIGM hyperIgM syndrome, HIES Hyper-IgE syndromes, IPEX immunodysregulation, polyendocrinopathy, enteropathy X-linked syndrome, SCID severe combined immune deficiency, WAS Wiskott-Aldrich syndrome, XLA X-linked agammaglobulinemia

difference among subgroups of PID according to the age of onset and age at the time of diagnosis (Table 2). Our study indicated that symptoms were notably observed earlier in WAS and SCID patients compared with other subgroups with the median onset age of 1 and 4 months, respectively; meanwhile, the time of the first diagnosis was shorter in WAS and SCID patients with the median diagnosis time of 7.22 and 5.94 months, respectively. Compared with WAS and SCID patients, patients with primary antibody deficiency displayed symptoms remarkably later with the median age of 26.5 months, in which the mean time to diagnosis was 41.8 months.

\section{Main clinical manifestations}

In our study population, 20 patients (17.8\%) had a positive family history of PID (Table 2). Additionally, patients with PID presented various clinical manifestations during diagnosis (Table 3). A previous history of recurrent infection was found in almost all the patients $(n=109,97.3 \%)$. The main pathogen of infection was bacterial infection $(n=108)$, and fungal infection $(n=18)$ ranked in the second place, followed by viral infection in the third place $(n=7)$. Respiratory infection was the most common complication $(n=89$, 79.5\%), including sinusitis, acute otitis, bronchitis, bronchiectasis, and pneumonia. The second common complications were infections of skin and mucous membranes. There were 38 cases of skin and mucous membrane infection (33.9\%), including 11 cases of mouth ulcers, 9 cases of perianal abscess, 7 cases of mycotic stomatitis, 7 cases of skin pustules and nodules, and 4 case of eczema. The third common complication was digestive tract infection, of which diarrhea was found in 26 cases (23.2\%). However, diarrhea not only is caused by infection, but also caused by immune disorder. In addition, we found 7 cases with infection of central nervous system (CNS). It should be noted that 23 children (20.5\%) had adverse event following immunization (AEFI), involving Bacillus Calmette-Guérin (BCG)-osis, local skin infection, and fever. Furthermore, 21 patients (17\%) had BCG-osis 
Table 2 General characteristics of patients with primary immunodeficiency diseases

\begin{tabular}{|c|c|c|c|c|c|c|c|c|}
\hline Category & $\begin{array}{l}\text { No. of } \\
\text { Cases }\end{array}$ & $\begin{array}{l}\text { Gender } \\
\text { Ratio (F/M) }\end{array}$ & $\begin{array}{l}\text { No. of } \\
\text { Death }\end{array}$ & $\begin{array}{l}\text { Age at death } \\
(\mathrm{mo})\end{array}$ & $\begin{array}{l}\text { No. of } \\
\text { HSCT }\end{array}$ & $\begin{array}{l}\text { Age at } \\
\text { onset (mo) }\end{array}$ & $\begin{array}{l}\text { Age at } \\
\text { diagnosis } \\
\text { (mo) }\end{array}$ & $\begin{array}{l}\text { Family } \\
\text { history }\end{array}$ \\
\hline SCID & 32 & $22 / 10$ & 30 & $\begin{array}{l}7.03 \pm 1.25 \\
(1-28)\end{array}$ & 7 & $\begin{array}{l}4.03 \pm 0.88 \\
(0.5-23)\end{array}$ & $\begin{array}{l}5.94 \pm 1.06 \\
(1-28)\end{array}$ & 7 \\
\hline HIGM & 27 & $27 / 0$ & 4 & $\begin{array}{l}88.50 \pm 44.66 \\
(16-205)\end{array}$ & 24 & $\begin{array}{l}9.536 \pm 2.23 \\
(1-60)\end{array}$ & $\begin{array}{l}31.25 \pm 6.23 \\
(3-120)\end{array}$ & 4 \\
\hline WAS & 9 & $9 / 0$ & 0 & & 9 & $\begin{array}{l}1.00 \pm 0.14 \\
(0.5-2)\end{array}$ & $\begin{array}{l}7.22 \pm 1.98 \\
(2-22)\end{array}$ & 0 \\
\hline DGS & 1 & $1 / 0$ & & & & 0.5 & 0.5 & 0 \\
\hline DKC & 2 & $2 / 0$ & 1 & 26 & 1 & $\begin{array}{l}37.00 \pm \\
23.00\end{array}$ & $44.00 \pm 20.00$ & 1 \\
\hline EDA-ID & 1 & $1 / 0$ & 1 & 4 & & 0.5 & 3 & 1 \\
\hline HIES & 1 & $1 / 0$ & 1 & 133 & & 60 & 60 & 0 \\
\hline $\begin{array}{l}\text { Severe reduction in all serum immunologlobulin } \\
\text { isotypes with profoundly decreased or absent B cells }\end{array}$ & 16 & $15 / 1$ & 3 & $\begin{array}{l}46.00 \pm 23.29 \\
(3-83)\end{array}$ & & $\begin{array}{l}26.50 \pm 6.89 \\
(2-60)\end{array}$ & $\begin{array}{l}41.80 \pm 10.44 \\
(3-120)\end{array}$ & 3 \\
\hline CVID & 3 & $3 / 0$ & 0 & & & $\begin{array}{l}29.33 \pm \\
13.13(4-48)\end{array}$ & $\begin{array}{l}46.33 \pm 26.21 \\
(7-96)\end{array}$ & 0 \\
\hline APDS & 1 & $1 / 0$ & 0 & & & 96 & 96 & 0 \\
\hline IPEX syndrome & 2 & $2 / 0$ & 0 & & 2 & 6,28 & 6,28 & 0 \\
\hline Immune dysregulation with colitis & 1 & $0 / 1$ & 1 & 13 & & 1 & 4 & 0 \\
\hline CHS & 1 & $0 / 1$ & 0 & & 1 & 2 & 68 & 0 \\
\hline Congenital neutropenias & 5 & $4 / 1$ & 0 & & 1 & & & 2 \\
\hline CGD & 7 & $6 / 1$ & 1 & 12 & 1 & $\begin{array}{l}2.17 \pm 1.17 \\
(1-8)\end{array}$ & $\begin{array}{l}5.00 \pm 2.11 \\
(1-15)\end{array}$ & 2 \\
\hline IFN- $\gamma$ receptor deficiency & 1 & $0 / 1$ & 0 & & 1 & 2 & 60 & 0 \\
\hline $\begin{array}{l}\text { Predisposition to invasive fungal diseases } \\
\text { (CARD9 deficiency) }\end{array}$ & 1 & $1 / 0$ & 0 & & & 117 & 130 & 0 \\
\hline Complement deficiencies & 1 & $1 / 0$ & 0 & & & 48 & 60 & 0 \\
\hline
\end{tabular}

Abbreviations: APDS activated PI3K $\delta$ syndrome immunodeficiency, CGD chronic granulomatous disease, CHS Chediak-Higashi syndrome, CVID common variable immunodeficiency disorders, DGS DiGeorge syndrome, DKC dyskeratosis congenital, EDA-ID anhidrotic ectodermodysplasia with immunodeficiency, HIGM hyperIgM syndrome, HIES Hyper-lgE syndromes, IPEX immunodysregulation, polyendocrinopathy, enteropathy X-linked syndrome, SCID severe combined immune deficiency, WAS Wiskott-Aldrich syndrome, XLA X-linked agammaglobulinemia

after BCG vaccination, and BCG-osis was mainly observed in patients with SCID (9 cases), chronic granulomatous disease (7 cases), idiopathic primary hypogammaglobulinemia (4 cases), and interferon-gamma receptor deficiency (1 case). Thus, AEFI may lead to the consideration of the possibility of PID. Additionally, a variety of clinical manifestations, such as leukopenia, anemia, thrombocytopenia, seizure, rash, and lymphoma, were rarely observed in the patients with PID in our study.

\section{Outcomes}

Of the 112 patients, 43 (33 males and 10 females, 38.4\%) died due to recurrent and severe infections during the follow-up. The mean age of death for SCID patients was 7 months, while patients with HIGM syndrome and predominantly antibody deficiencies had the mean age of death equal to 88.5 and 46 months, respectively (Table 2). Of these, 38 died during hospitalization, and 5 died after discontinuing treatment (all with SCID). In addition, 65 patients survived, and 4 patients were lost follow-up during the follow-up period. Respiratory failure after pneumonia, sepsis, complications after allogeneic hematopoietic stem cell transplantation (allo-HSCT), disseminated BCG, and pericardial effusion were the most common causes of death as well. It is noteworthy that $93.8 \%(30 / 32)$ of the SCID patients died (including 6 cases who underwent allo-HSCT) except for 1 case who underwent allo-HSCT and the other who is currently alive and preparing to undergo alloHSCT. In the present study, a total of 47 cases underwent allo-HSCT, and the 2-year overall survival (OS) rate was 78.7\%. The OS widely differed among PID patients with different phenotypes who underwent allo-HSCT (Table 4). Among 7 patients with SCID who underwent allo-HSCT, 3 patients died of sepsis, 2 patients died of graft-versushost-disease (GVHD), and 1 patient died of pretreatment before allo-HSCT, while only 1 case was survived. Among 24 patients with HIGM syndrome who underwent alloHSCT, 3 patients died of sepsis, 1 patient died of GVHD, 
Table 3 Clinical manifestations and complications of patients with primary immunodeficiency diseases

\begin{tabular}{|c|c|c|c|c|c|c|}
\hline Type & $\begin{array}{l}\text { Respiratory } \\
\text { infections }\end{array}$ & $\begin{array}{l}\text { Repeated } \\
\text { Diarrhea }\end{array}$ & $\begin{array}{l}\text { Bacterial Infection Of Skin } \\
\text { and Mucous Membrane }\end{array}$ & $\begin{array}{l}\text { Fungal } \\
\text { Infection }\end{array}$ & Meningitis & BCG-osis \\
\hline$\overline{S C I D}$ & 19 & 13 & 8 & 6 & 4 & 9 \\
\hline HIGM & 26 & 6 & 10 & 7 & 0 & 4 \\
\hline WAS & 5 & 2 & 4 & 0 & 0 & 0 \\
\hline DGS & 1 & 0 & 0 & 0 & 0 & 0 \\
\hline DKC & 2 & 1 & 1 & 0 & 0 & 0 \\
\hline EDA-ID & 1 & 0 & 0 & 0 & 0 & 0 \\
\hline HIES & 1 & 0 & 0 & 0 & 0 & 0 \\
\hline $\begin{array}{l}\text { Severe reduction in all serum immunologlobulin isotypes } \\
\text { with profoundly decreased or absent B cells }\end{array}$ & 14 & 1 & 2 & 1 & 2 & 0 \\
\hline CVID & 3 & 0 & 0 & 0 & 1 & 0 \\
\hline APDS & 1 & 0 & 0 & 0 & 0 & 0 \\
\hline IPEX syndrome & 1 & 2 & 1 & 1 & 0 & 0 \\
\hline Immune dysregulation with colitis & 0 & 1 & 1 & 0 & 0 & 0 \\
\hline $\mathrm{CHS}$ & 0 & 0 & 1 & 0 & 0 & 0 \\
\hline Congenital neutropenias & 7 & 0 & 1 & 2 & 1 & 0 \\
\hline CGD & 6 & 1 & 6 & 0 & 0 & 7 \\
\hline IFN- $\gamma$ receptor deficiency & 1 & 0 & 1 & 0 & 0 & 1 \\
\hline CARD9 deficiency & 0 & 0 & 1 & 1 & 1 & 0 \\
\hline Complement deficiencie & 1 & 0 & 1 & 0 & 1 & 0 \\
\hline Total (\%) & $89(79.5)$ & $26(23.2)$ & $38(33.9)$ & $18(16.1)$ & $10(8.9)$ & $21(18.8)$ \\
\hline
\end{tabular}

and 20 cases were survived. The remaining cases were all survived and exhibited complete immune reconstitution. The 2-year OS rate for patients with SCID, HIGM syndrome, and the remaining patients with PID who received allo-HSCT was 14.3, 83.3, and 100\%, respectively (Table 4).

\section{Discussion}

PIDs are rare inherited diseases involved the immune system, typically associating with recurrent and severe infection, autoimmune disease and increased incidences of

Table 4 The overall survival of PIDs patients after allogeneic HSCT

\begin{tabular}{llll}
\hline Type & No. of Cases & No. of Survival & No. of Death \\
\hline Total & 47 & 37 & 10 \\
SCID & 7 & 1 & 6 \\
HIGM & 24 & 20 & 4 \\
WAS & 9 & 9 & 0 \\
DKC & 1 & 1 & 0 \\
IPEX syndrome & 2 & 2 & 0 \\
CHS & 1 & 1 & 0 \\
Congenital neutropenias & 1 & 1 & 0 \\
CGD & 1 & 1 & 0 \\
IFN- $-\gamma$ receptor deficiency & 1 & 1 & 0 \\
\hline
\end{tabular}

malignancies. At present, fast diagnosis of PID in nonspecialized hospitals or clinics across China accompanies with great challenges. Delayed diagnosis and misdiagnosis commonly occur, mainly leading to poor clinical prognosis. In the current research, we studied 112 patients with PID during 6 years in our hospital based on their clinical, immunological, and molecular characteristics, aiming to provide a reference for perfect diagnosis of PID.

In the present research study, the most common PID was combined immunodeficiencies (28.6\%), followed by HIGM syndrome $(24.1 \%)$, predominantly antibody-deficiency diseases (17.8\%), combined immunodeficiencies with associated or syndromic features $(12.5 \%)$, congenital defects of phagocyte number or function (10.7\%), diseases of immune dysregulation (3.6\%), defects in intrinsic and innate immunity $(1.8 \%)$, and complement deficiencies accounted for about $0.9 \%$ of cases, which were found to be different from those previously reported [6-8] probably because our hospital is a tertiary referral center and our cases were inpatients and a number of them were admitted from elsewhere. Therefore, the proportion of patients with PID, who are prone to have severe complications and difficult to diagnose, significantly increased. In addition, our hospital is one of the main referral centers for allo-HSCT in China, and some of the PID patients in our study were previously diagnosed with PID in other hospitals who needed to receive 
allo-HSCT. Furthermore, we cannot ignore a possibility that a number of differences were based on race and relative incidence of disease in our population. Our data further showed that the male/female ratio was $6.0: 1$, and $60.7 \%$ of the cases presented symptoms less than 6 months. Besides, $17.8 \%$ of the patients in our study had family history of PID, which was found similar to a previous study [8].

Repeated and chronic infections, particularly pulmonary infections, are a main feature of PID. Large sample cohort studies on PID indicated that almost all patients with PID had a history of recurrent infection before diagnosis was finalized [9-11]. In agreement with those reports, the present study revealed that $97.3 \%$ of PID patients presented recurrent infections before diagnosis. The most common occurrences were respiratory tract infection, followed by bacterial infection of the skin and mucous membranes in 38 cases (33.9\%). Of note, gastrointestinal disorders are frequent in patients with PID $[12,13]$. Moreover, 26 (112, 23.2\%) patients with infectious or noninfectious diarrhea showed a poor growth as well. Besides, PID-related gastrointestinal diseases not only are caused by infection, but also by autoimmunity, an inflammatory response, or malignancy. Recurrent gastrointestinal symptom could be the first presentation of PID, thus physicians should be aware of the possibility of PID in patient with intractable diarrhea, malabsorption, and failure to thrive, especially those cases who failed to respond to conventional treatment strategies $[14,15]$.

Sarmiento et al. reported $7.65 \%$ of AEFI in patients with PID [16]. The majority of cases of AEFI have occurred in patients with CGD, SCID, and idiopathic primary hypogammaglobulinemia. It was reported that BCG is a vaccine, mainly associating with AEFI in patients with PID [17]. In the current study, AEFI was found in $20.5 \%$ of patients with PID after vaccination, especially with BCG vaccine, and included extra regional lymph nodes, skin, or lungs as the most common clinical presentations. For patients with CGD and SCID, the percentage of BCG-osis was 100 and $28.1 \%$, respectively, which found to be in line with previously reported findings [18-20]. Notably, $17.8 \%$ of the cases included family history of PID. Because BCG vaccination is routinely carried out at birth in China, PID patients who receive BCG vaccination before immune deficiency are highly suspected. BCG vaccination should be avoided if any family history or clinical or laboratory evidence concerns a neonate's immune competency. Moreover, disseminated BCG infection should be suspected in any vaccinated infants who accompany with a persistent fever or comparable disease of unknown etiology.

In addition to recurrent and severe infections, immunity disorder is commonly observed in PID patients, especially in patients who accompany with predominantly antibody deficiencies [21, 22]. A recent study, that involved an Iranian cohort of 471 patients, reported inflammatory manifestations in $26.5 \%$ of patients. Furthermore, the prevalence of immunity disorder appeared to increase with age in PID cohorts, influencing a significant proportion of patients [23]. In this study, we also found that not only autoimmune gastrointestinal disease, but also autoimmune cytopenias were the common autoimmune manifestations in patients with PID. When a child associates with autoimmune manifestations, the possibility of incidence of PID should be highly taken into account.

Immunoglobulin replacement therapy and allo-HSCT are effective therapeutic strategies for patients with PID. On the basis of previous reports, immunoglobulin replacement therapy was found to be effective for $83 \%$ of children with hypogammaglobulinemia [24, 25]. At present, the majority of patients with PID only receive symptomatic treatment. Allo-HSCT, which has been used as an effective treatment for PID [26, 27], isn't frequent in China. The 2 -year OS rate in the SCID patients was $90 \%$ during 25 months of follow-up [28]. A total of 47 cases underwent allo-HSCT and the OS rate was $78.7 \%$ (37/47) in the present study, which was in agreement with those findings previously reported [27]. However, the OS widely differed among PID patients with different phenotypes who underwent allo-HSCT. The 2-year OS rate for SCID, HIGM syndrome, and the remaining of PID patients who underwent allo-HSCT was 14.3, 83.3, and 100\%, respectively. The OS of SCID is mainly poor because SCID patients are often severely infected, and bone marrow transplantation of severely infected SCID children has a poor prognosis. Compared with patients with active infection and older age who underwent allo-HSCT, OS was better in those patients who received a transplant when they were younger and free from infection. Thus, allo-HSCT is more effective in younger PID children, and allo-HSCT is often helpful when an appropriate donor is available.

However, the present study contains some limitations, including its retrospective nature, small sample size, and all the cases were recruited at a single center. A possible selection bias also might be present because the clinical data were taken from hospitalized children with PID. Further researches involving more PID patients and multiple centers are required in the future study.

\section{Conclusions}

Our study indicated that PID typically emerges at early age. Recurrent infection and serious infection were the most common clinical manifestations. Delayed diagnosis or misdiagnosis may lead to poor clinical prognosis as well. Allo-HSCT is a relatively effective therapeutic strategy for PID patients. Our study may provide a reliable reference for pediatricians to diagnosis children with PID. 


\section{Abbreviations}

AEFI: adverse events following immunization; Allo-HSCT: allogeneic hematopoietic stem cell transplantation; APDS: activated PI3K syndrome immunodeficiency; BCG: Bacillus Calmette-Guérin; CGD: chronic granulomatous disease; CHS: Chediak-Higashi syndrome; CVID: common variable immunodeficiency disorders; DGS: DiGeorge syndrome; DKC: dyskeratosis congenital; EDA-ID: anhidrotic ectodermodysplasia with immunodeficiency; GVHD: graft versus host disease; HIES: Hyper-lgE syndromes; HIGM: hyper-IgM syndrome; IPEX: immunodysregulation, polyendocrinopathy, enteropathy X-linked syndrome; IUIS: International Union of Immunological Societies; OS: overall survival; PIDs: primary immunodeficiency diseases; SCID: severe combined immune deficiency; WAS: Wiskott-Aldrich syndrome; XLA: X-linked agammaglobulinemia

\section{Acknowledgments}

None.

\section{Authors' contributions}

$\mathrm{JH}-\mathrm{W}$ designed the study, wrote the first and final drafts, and revised the manuscript. $W W-Z$ and $Y Y$ analyzed the data and conducted statistical analyses. WW-Z and JH-W prepared Figs. HZ provided critical feedback. All authors read and approved the final manuscript.

\section{Funding}

This work was supported by grants from the National Natural Science Foundation of China (81400019, to Jinhong Wu), the Science and Technology Commission Foundation of Shanghai Pudong New Area (No. PKJ2013-Y65, to Jinhong Wu) and the Shanghai Science and Technology Commission Biomedicine Department key Science and Technology Projects (16411950800, to Hao Zhang).

\section{Availability of data and materials}

The datasets used in the current study are available from the corresponding author upon reasonable request.

\section{Ethics approval and consent to participate}

The Ethics Committee of the Shanghai Children's Medical Center Affiliated to Shanghai Jiaotong University School of Medicine approved the study, and each patient provided written informed consent.

\section{Consent for publication}

Not applicable.

\section{Competing interests}

The authors declare that they have no competing interests.

\section{Received: 24 March 2019 Accepted: 20 September 2019}

\section{Published online: 04 November 2019}

\section{References}

1. Picard C, Bobby Gaspar H, Al-Herz W, et al. International Union of Immunological Societies: 2017 primary immunodeficiency diseases committee report on inborn errors of immunity. J Clin Immunol. 2018; 38:96-128.

2. Zhang ZY, An YF, Jiang LP, Liu W, Liu DW, Xie JW, Tang XM, Wang M, Yang $X Q$, Zhao XD. Distribution, clinical features and molecular analysis of primary immunodeficiency diseases in Chinese children: a single-center study from 2005 to 2011. Pediatr Infect Dis J. 2013:32(10):1127-34.

3. Zeng $H$, Tao $Y$, Chen $X$, et al. Primary immunodeficiency in South China: clinical features and a genetic subanalysis of 138 children. J Investig Allergol Clin Immunol. 2013;23(5):302-8.

4. Bousfiha A, Jeddane L, Picard C, et al. The 2017 IUIS phenotypic classification for primary Immunodeficiencies. J Clin Immunol. 2018;38:129-43.

5. Picard C, Al-Herz W, Bousfiha A, et al. Primary immunodeficiency diseases: an update on the classification from the International Union of Immunological Societies Expert Committee for primary immunodeficiency 2015. J Clin Immunol. 2015;35:696-726.

6. Gathmann B, Binder N, Ehl S, et al. The European internet-based patient and research database for primary immunodeficiencies: update 2011. Clin Exp Immunol. 2012;167(3):479-91.
7. Kobrynski L, Powell RW, Bowen S. Prevalence and Morbidity of Primary Immunodeficiency Diseases, United States 2001-2007. J Clin Immunol. 2014 Nov;34(8):954-61.

8. Rubin Z, Pappalardo A, Schwartz A, Antoon JW. Prevalence and Outcomes of Primary Immunodeficiency in Hospitalized Children in the United States. J Allergy Clin Immunol Pract. 2018 Sep - Oct:6(5):1705-10.

9. Modell V, Quinn J, Orange J, Notarangelo LD, Modell F. Primary immunodeficiencies worldwide: an updated overview from the Jeffrey Modell centers global network. Immunol Res. 2016;64:736-53.

10. Reisi M, Azizi G, Kiaee F, et al. Evaluation of pulmonary complications in patients with primary immunodeficiency disorders. Eur Ann Allergy Clin Immunol. 2017;49(3):122-8.

11. Barlogis V, Mahlaoui N, Auquier P, et al. Burden of poor health conditions and quality of life in 656 children with primary immunodeficiency. J Pediatr. 2018:194:211-7.

12. Agarwal S, Mayer L. Diagnosis and treatment of gastrointestinal disorders in patients with primary immunodeficiency. Clin Gastroenterol Hepatol. 2013;11:1050-63.

13. Pecoraro A, Nappi L, Crescenzi L, D'Armiento FP, Genovese A, Spadaro G. Chronic diarrhea in common variable immunodeficiency: a case series and review of the literature. J Clin Immunol. 2018;38:67-76.

14. Angelino G, De Angelis $P$, Faraci $S$, et al. Inflammatory bowel disease in chronic granulomatous disease: an emerging problem over a twenty years' experience. Pediatr Allergy Immunol. 2017;28:801-9.

15. Barzaghi F, Amaya Hernandez LC, Neven B, et al. Long-term follow-up of IPEX syndrome patients after different therapeutic strategies: an international multicenter retrospective study. J Allergy Clin Immunol. 2018;141:1036-49.

16. Sarmiento JD, Villada F, Orrego JC, Franco JL, Trujillo-Vargas CM. Adverse events following immunization in patients with primary immunodeficiencies. Vaccine. 2016;34:1611-6.

17. Bonilla FA. Update: vaccines in primary immunodeficiency. J Allergy Clin Immunol. 2018;141:474-81.

18. Marciano $B E$, Huang $C Y$, Joshi $G$, et al. BCG vaccination in patients with severe combined immunodeficiency: complications, risks, and vaccination policies. J Allergy Clin Immunol. 2014;133:1134-41.

19. Norouzi S, Aghamohammadi A, Mamishi S, Rosenzweig SD, Rezaei N. Bacillus Calmette-Guérin (BCG) complications associated with primary immunodeficiency diseases. J Inf Secur. 2012;64:543-54.

20. Al-Hammadi S, Alsuwaidi AR, Alshamsi ET, Ghatasheh GA, Souid AK. Disseminated Bacillus Calmette-Guérin (BCG) infections in infants with immunodeficiency. BMC Res Notes. 2017:10:177.

21. Schmidt RE, Grimbacher B, Witte T. Autoimmunity and primary immunodeficiency: two sides of the same coin? Nat Rev Rheumatol. 2017;14:7-18

22. Susheela AT, Hale A. Patients with common variable immunodeficiency paradoxically have increased rates of autoimmune disorders. BMJ Case Rep. 2017.

23. Azizi $\mathrm{G}$, Tavakol M, Rafiemanesh $\mathrm{H}$, et al. Autoimmunity in a cohort of 471 patients with primary antibody deficiencies. Expert Rev Clin Immunol. 2017; 13:1099-106.

24. Bienvenu B, Cozon G, Hoarau C, et al. Does the route of immunoglobin replacement therapy impact quality of life and satisfaction in patients with primary immunodeficiency? Insights from the French cohort "Visages.". Orphanet J Rare Dis. 2016:11:83.

25. Krivan G, Jolles S, Granados EL, et al. New insights in the use of immunoglobulins for the management of immune deficiency (PID) patients. Am J Clin Exp Immunol. 2017;6(5):76-83.

26. Slatter MA, Gennery AR. Hematopoietic cell transplantation in primary immunedeficienc-y-conventional and emerging indications. Expert Rev Clin Immunol. 2018:14:103-14.

27. Fox TA, Chakraverty R, Burns S, et al. Successful outcome following allogeneic hematopoietic stem cell transplantation in adults with primary immunodeficiency. Blood. 2018;131:917-31.

28. Heimall J, Logan BR, Cowan MJ, et al. Immune reconstitution and survival of 100 SCID patients post-hematopoietic cell transplant: a PIDTC natural history study. Blood. 2017;130:2718-27.

\section{Publisher's Note}

Springer Nature remains neutral with regard to jurisdictional claims in published maps and institutional affiliations. 